14 Al-Ali H-K, Heinrich MC, Lange T, Krahl R, Mueller M, Müller C et al. High incidence of BCR-ABL kinase domain mutations and absence of mutations of the PDGFR and KIT activation loops in CML patients with secondary resistance to imatinib. Hematol J 2004; 5: 55-60.

15 Redaelli S, Mologni L, Rostagno R, Piazza R, Magistroni V, Ceccon M et al. Three novel patient-derived $B C R / A B L$ mutants show different sensitivity to second and third generation tyrosine kinase inhibitors. Am J Hematol 2012; 87: E125-E128.

16 Zhou T, Commodore L, Huang W-S, Wang Y, Thomas M, Keats J et al. Structural mechanism of the pan-BCR-ABL inhibitor ponatinib (AP24534): Lessons for overcoming kinase inhibitor resistance. Chem Biol Drug Des 2011; 77: $1-11$.

Supplementary Information accompanies this paper on the Leukemia website (http://www.nature.com/leu)

\title{
OPEN
}

\section{CD4+ T cells, but not non-classical monocytes, are dispensable for the development of chronic lymphocytic leukemia in the TCL1-tg murine model}

Leukemia (2016) 30, 1409-1413; doi:10.1038/leu.2015.307

In the adaptive immune system, crosstalk between B cells and T cells is pivotal to the expansion, differentiation, survival and effector functions of normal B cells in response to antigen. Chronic lymphocytic leukemia (CLL) is characterized by the accumulation of $\mathrm{CD}^{+} \mathrm{CD} 19^{+} \mathrm{B}$ cells in the peripheral blood, secondary lymphoid organs and bone marrow of patients. Although the cellular origin of CLL cells is still a subject of debate, molecular profiling demonstrates that CLL cells share similar signatures with antigen-experienced memory B cells. In this regard, it is believed that T-cell-dependent mechanisms similar to those required during the normal maturation of B cells may also operate in CLL and contribute to disease progression. We recently demonstrated that the accumulation of clonal CD4+ T cells in CLL is associated with B-cell receptor (BCR) characteristics (mutation status and stereotypy), implicating specific T-cell crosstalk with CLL cells during tumor outgrowth. ${ }^{1}$ This notion is further supported by the observation that so-called lymphoid proliferation centers, which are structural hallmarks in CLL, are composed of different antigen-presenting cells and numerous $\mathrm{CD} 4^{+}$ $\mathrm{T}$ cells that are clustered together with activated CLL cells. ${ }^{2}$ CLL activation in turn leads to enhanced viability, chemoresistance, and proliferation. Indeed, both in vitro and in vivo data demonstrate that activated $\mathrm{CD}^{+} \mathrm{T}$ cells provide stimuli, for example, CD40L, that drive CLL cells into proliferation. ${ }^{3,4}$ In addition, $\mathrm{CD}^{+} \mathrm{T}$ cells can protect CLL cells from spontaneous apoptosis in vitro. ${ }^{5}$ As such, reversing the nature of CLL-T-cell interactions with immunomodulatory drugs is a therapeutic goal in CLL. ${ }^{6,7}$

To clarify the exact nature of $\mathrm{CD}^{+} \mathrm{T}$ cell help in the pathogenesis of CLL, we made use of GK5 mice (C57BL/6 background, hereafter referred to as GK mice), ${ }^{8}$ which exhibit a complete loss of $\mathrm{CD}^{+} \mathrm{T}$ cells in the periphery (Supplementary Figure $1 \mathrm{~A}$ ) due to the expression of a CD4-targeting antibody, in conjunction with the TCL1-tg mouse (C57BL/6 background), which currently represents the best validated mouse model for human CLL, and has been shown to recapitulate some of the T-cell abnormalities associated with the disease. ${ }^{9,10}$ Established tumors derived from spleens of leukemic TCL1-tg mice were adoptively transferred intraperitoneally into GK recipients or their wild-type (WT) littermate controls to assess the impact of the loss of CD4+ cells on the outgrowth of established malignant clones. Surprisingly, CLL tumors developed faster in GK mice compared with WT recipients (median latency 71 vs 177 days, $P=0.0294$; Figure 1a, left), suggesting that at least a subset of CD4+ T cells may serve to hinder tumor growth. We next studied the role of $\mathrm{CD}^{+} \mathrm{T}$ cells in the establishment of primary disease, as it is expected that CD4+ $T$ cells have a role in the initial selection and expansion of the malignant clone. We therefore crossed GK mice with TCL1-tg mice and monitored the development of CLL in all genotypes generated by this breeding (WT, GK, TCL1-tg and GK/TCL1-tg). Neither WT nor GK mice developed CLL. However, we again observed that tumors developed with significantly faster kinetics in the absence of $\mathrm{CD}^{+}$cells (GK/TCL1-tg mice) compared with TCL1-tg littermates (median latency 417 days vs 443 days, $P=0.0276$; Figure $1 \mathrm{a}$, right). The extent of splenomegaly (Figure $1 \mathrm{~b}$ ) and degree of tumor cell infiltration into different organs at the end point of the disease (Figure 1c) was similar in the absence or presence of CD4+ cells. In addition, to verify that a mono- or oligoclonal disease had established in GK/TCL1-tg mice, or to determine whether the lack of $\mathrm{CD}^{+}$cells fostered the outgrowth of a polyclonal tumor, we assessed the clonality of the BCR in tumor cells that developed in GK/TCL1-tg mice and TCL1-tg littermate controls. BCR spectratyping analyses (Supplementary Methods) confirmed that mono- and oligoclonal tumors developed in the absence of $\mathrm{CD}^{+}$cells to the same degree as in TCL1tg mice (Figure 1d and Supplementary Figure 1B). Thus, the data demonstrate that $\mathrm{CD} 4^{+} \mathrm{T}$ cell help is not absolutely essential to the establishment and progression of CLL in mice. This challenges the current view of CLL pathogenesis and indicates that other immune subsets, such as those derived from the myeloid lineage, may substitute in supporting CLL development in this model.

Flow cytometric analyses of peripheral blood leukocytes in GK mice (Supplementary Figure 2) revealed that absolute numbers of circulating monocytes were increased in GK mice compared with controls, an observation not previously published with the characterization of these mice. ${ }^{8}$ These data are relevant given that monocytes represent an important supportive immune subset within the CLL micromillieu that significantly impacts disease prognosis. CLL patients have a higher absolute number of circulating monocytes and a significant increase in non-classical monocytes $\left(\mathrm{CD} 14^{+} \mathrm{CD} 16^{++}\right)$compared with healthy controls. ${ }^{11}$ Moreover, Seiffert et al. ${ }^{12}$ established monocyte-derived soluble CD14 levels (sCD14) as a survival factor in CLL, and recently reported a skewing of splenic monocytes in TCL1-tg mice. ${ }^{7}$ We therefore analyzed peripheral blood cells of leukemic mice in 

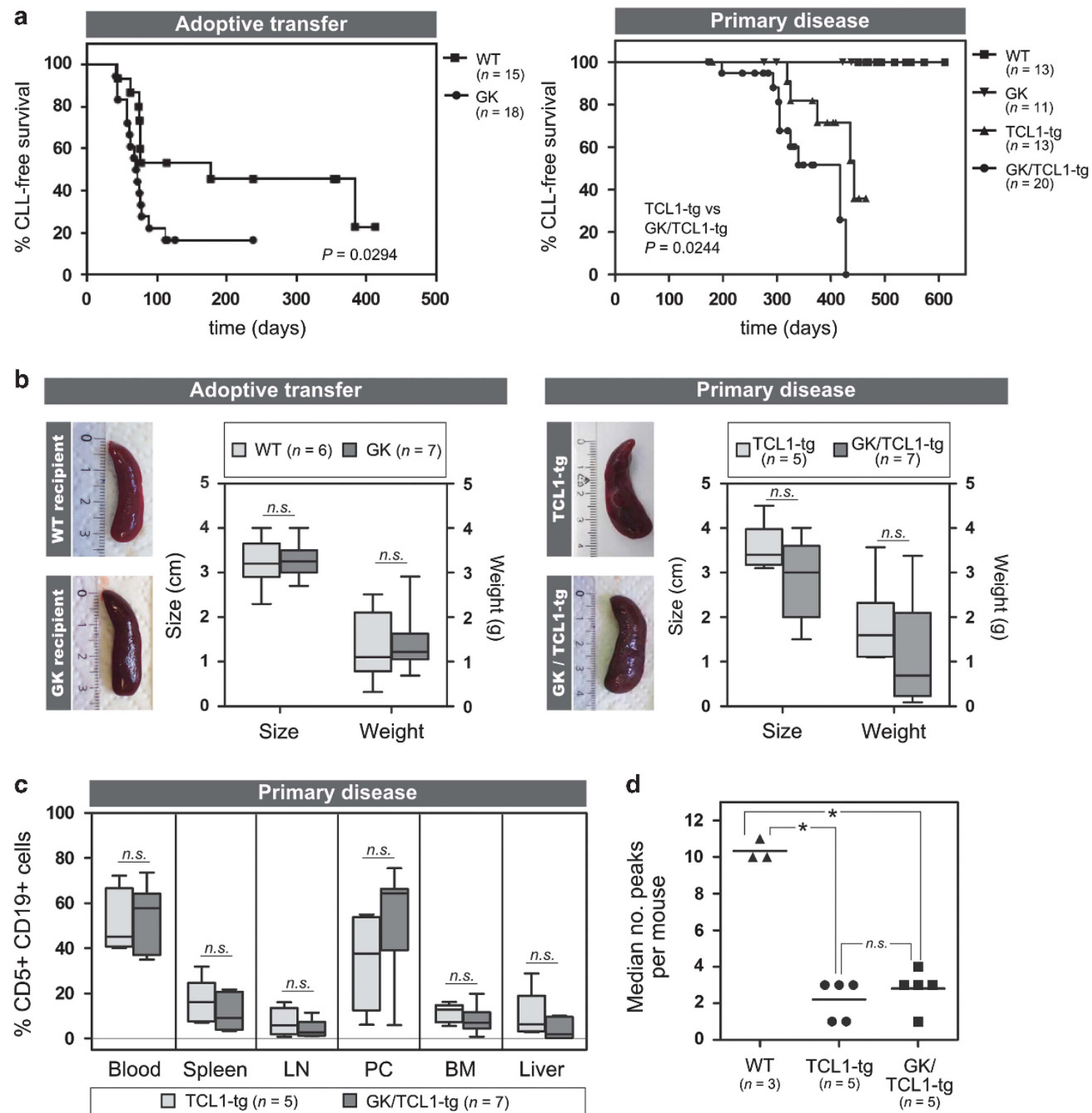

Figure 1. CD4+ T cells are not required for the establishment of CLL in the TCL1-tg murine model of the disease. For adoptive transfer experiments, splenocytes derived from spleens of leukemic TCL1-tg mice (10-20 $\times 10^{6}$ cells) were injected intraperitoneally into 6-8-weekold GK mice $(n=18)$ and their wild-type (WT) littermate controls $(n=15)$. To study the establishment of CLL in a primary disease model, TCL1-tg mice were crossed with GK mice to yield double-transgenic GK/TCL1-tg mice. Development of CLL was monitored in the blood at regular intervals in all cohorts (WT, $n=13 ; \mathrm{GK}, n=11$; TCL1-tg, $n=13 ; \mathrm{GK} / \mathrm{TCL} 1-\mathrm{tg}, n=20$ ). In both adoptive transfer and primary disease models, death was scored as a CLL event based on the presence of $>5000$ CD5+CD19+ cells per $\mu$ l blood or when these cells represented the most prominent lymphocyte fraction in at least three out of five lymphoid compartments examined. Loss of CD4+ T cells leads to decreased survival (Kaplan-Meier) in both adoptive transfer (left, median latency 71 days in GK vs 177 days in WT recipients, $P=0.0294$ ) and primary disease models (right, median latency 417 days in GK/TCL1-tg vs 443 days in TCL1-tg mice, $P=0.0276$ ). (b) The extent of splenomegaly in leukemic mice with or without CD4+ T cells was similar in both adoptive transfer (left panel: leukemic WT recipients, $n=6$; leukemic GK recipients, $n=7$ ) and primary disease (right panel: TCL1-tg, $n=5$; GK/TCL1-tg, $n=7$ ) models. (c) Single-cell suspensions of various organs collected upon killing of leukemic mice were analyzed by flow cytometry for the presence of CD5+CD19+ cells. The extent of tumor cell infiltration in the indicated organs is reported as the percentage of CD5+CD19+ cells of the total cell population. No significant differences could be observed between TCL1-tg $(n=5)$ and GK/TCL1-tg mice $(n=7)$ at the end stage of disease. (d) The clonality of the tumors in TCL1-tg $(n=5)$ and GK/TCL1-tg $(n=5)$ mice were assessed by BCR spectratyping analysis on CD5+ MACS-sorted (Miltenyi Biotec, Bergisch Gladbach, Germany) splenic cells and compared with CD19+ splenic B cells derived from WT mice $(n=3)$. ${ }^{*} P<0.05$; n.s., not significant.

order to characterize the various monocyte subsets in murine CLL. We defined murine classical monocytes as $\mathrm{Gr} 1(\mathrm{Ly} 6 \mathrm{C})^{++} \mathrm{CD}^{+} 3^{+}$and non-classical monocytes as $\mathrm{Gr} 1(\mathrm{Ly} 6 \mathrm{C})^{+} \mathrm{CD} 43^{++}$(Supplementary Figure $3 \mathrm{~A}$ ) as proposed by Ziegler-Heitbrock et al. ${ }^{13}$ Absolute monocyte numbers were increased in leukemic mice compared with controls (primary disease: TCL-tg vs WT, $P<0.0001$; GK/TCL1tg vs WT, $P<0.0001$; adoptive transfer: WT leukemic vs pre-engraftment, $P=0.001$; GK leukemic vs pre-engraftment, $P=0.0002$ ), but were not significantly different between the leukemic cohorts (Figure 2a). In line with observations in human 

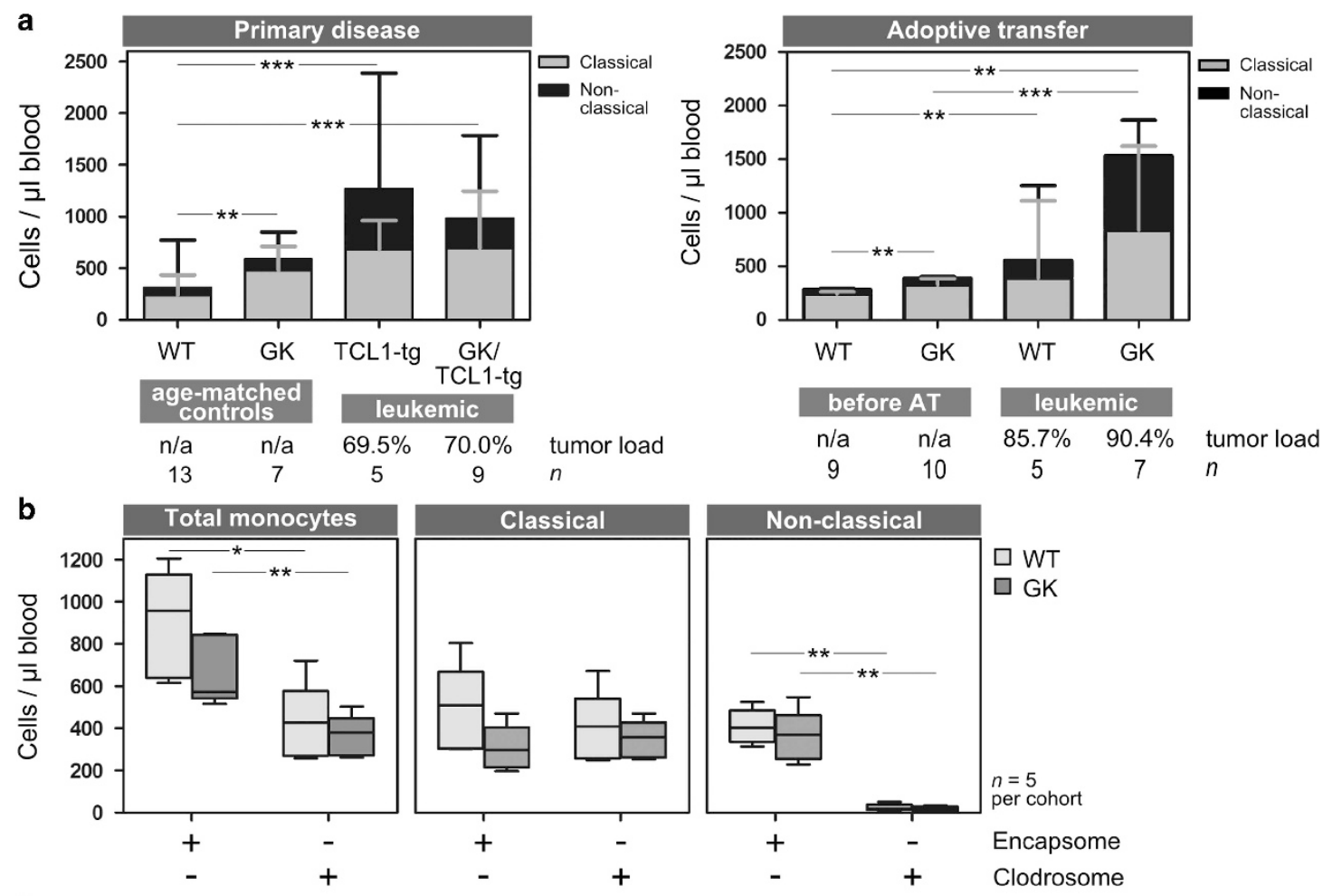

C
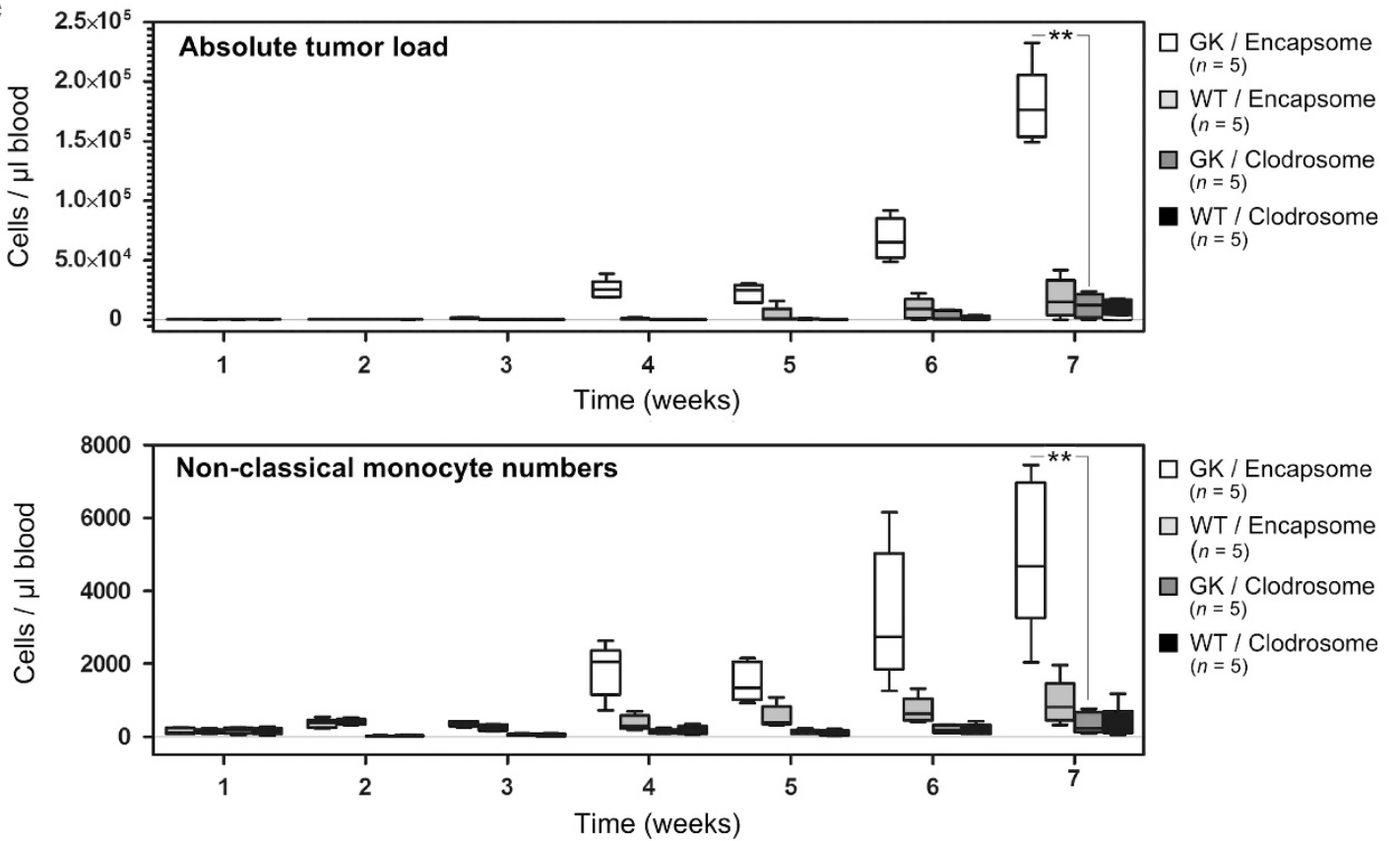

Figure 2. Impact of circulating monocytes on murine CLL development. (a) The absolute number of classical and non-classical monocytes were measured according to their surface expression of CD14, Gr1 and CD43 in the peripheral blood of leukemic mice at the end stage of disease in both primary disease (right panel) and adoptive transfer (left panel) models. For each cohort, the median tumor load (\% CD5+CD19+ of total lymphocytes) is given. In the primary disease model, circulating total monocyte numbers are increased in TCL1-tg ( $n=5)$ and GK/TCL1-tg $(n=9)$ mice compared with age-matched littermate controls (WT, $n=13 ; \mathrm{GK}, n=7)$ (left panel; TCL-tg vs WT, $P<0.0001 ; \mathrm{GK} / \mathrm{TCL} 1$-tg vs WT, $P<0.0001)$. Similarly, in the adoptive transfer model, total monocyte numbers are increased at the end stage of disease in both GK mice $(n=7)$ and WT littermates $(n=5)$, compared with pre-engraftment levels $(n=8$ and 7, respectively) (right panel; WT pre-adoptive transfer vs leukemic $P=0.001$; GK pre-adoptive transfer vs leukemic, $P=0.0002)$. (b) Treatment of both GK mice and their WT littermate controls with clodronate-loaded liposomes (Clodrosome) resulted in the selective depletion of non-classical monocytes compared with treatment with control liposomes (Encapsome). WT mice treated with Encapsome $(n=5)$ vs Clodrosome $(n=5), P<0.0001$; GK treated with Encapsome $(n=5)$ vs Clodrosome $(n=5)$, $P=0.0002$. The depletion of the non-classical monocyte fraction resulted in a reduction in total circulating monocyte numbers (WT Encapsome vs Clodrosome, $P=0.0095$; GK Encapsome vs Clodrosome, $P=0.0067)$. (c) Concomittant with the depletion of non-classical monocytes, tumor development was significantly delayed in engrafted WT or GK mice that had been treated with Clodrosome (top panel; GK Encapsome vs Clodrosome at week 7, $P<0.0001$ ) and the onset of tumor development paralleled the recovery of the circulating non-classical monocytes subset (bottom panel; GK Encapsome vs Clodrosome at week 7, $P=0.0011$ ). ${ }^{*} P<0.05$, ${ }^{* *} P<0.01$ and ${ }^{* * *} P<0.001$; n.s., not significant. 
CLL, TCL1-tg and GK/TCL1-tg leukemic mice showed significantly enhanced numbers of non-classical monocytes compared with age-matched littermate controls (Figure 2a, left panel: TCL1-tg vs WT, $P=0.0031 ;$ GK/TCL1-tg vs WT, $P=0.0011 ;$ TCL1-tg vs GK, $P=0.0025 ; G K / T C L 1-\operatorname{tg}$ vs $G K, P=0.0115)$. Similarly, in the adoptive transfer model, circulating non-classical monocytes were increased at the end stage of disease compared with their levels before tumor engraftment (Figure 2a, right panel: WT leukemic vs pre-engraftment, $P=0.001$; GK leukemic vs pre-engraftment, $P=0.0001$ ). These results demonstrate the striking parallels between human CLL and the TCL1-tg mouse model with regard to the monocyte population. Moreover, the data underscore the potential significance of tumor-supportive signals derived from monocytes in the establishment and progression of CLL, which may be especially important in the absence of CD4+ T cells. To confirm this, we exploited a method developed for specific macrophage/monocyte depletion using clodronate liposomes. ${ }^{14}$ Liposomal clodronate $(1 \mathrm{mg}$ Clodrosome, Encapsula Nanosciences LLC, Brentwood, TN, USA) or control liposomes (Encapsome, Encapsula Nanosciences LLC) were administered intraperitoneally into GK mice or WT littermates 1 day before adoptive transfer of tumor cells, and treatment continued for 2 weeks (every 3-4 days, 4 injections total; Supplementary Methods). Circulating monocyte numbers and subset distribution, as well as tumor load, were assessed by flow cytometry of peripheral blood samples drawn regularly over a period of 7 weeks. Clodrosome treatment resulted in the partial but significant depletion of circulating blood monocytes compared with treatment with control liposomes (WT Encapsome vs Clodrosome, $P=0.0095$; GK Encapsome vs Clodrosome, $P=0.0067$; Figure $2 \mathrm{~b}$ ). Interestingly, when analyzing monocyte subset distribution, it became clear that Clodrosome treatment led to the selective and significant reduction of the non-classical monocyte subset (WT Encapsome vs Clodrosome, $P<0.0001$; GK Encapsome vs Clodrosome, $P=0.0002$; Figure $2 \mathrm{~b}$ and Supplementary Figure $3 \mathrm{~B}$ ). Importantly, Clodrosome treatment effectively delayed tumor development in engrafted mice (Figure 2c, top), which was particularly striking in GK recipients (GK Clodrosome vs Encapsome at week $7, P<0.0001$ ), presumably owing to the loss of the supportive non-classical monocytic subset. Consistent with this theory, tumor development after the end of Clodrosome treatment was accompanied by the recovery and/or outgrowth of the non-classical monocyte subset in Clodrosome- and Encapsome-treated mice (GK Clodrosome vs Encapsome at week 7, $P=0.0011$; Figure 2c and Supplementary Figure $3 C$ ). Indeed, we could demonstrate that the absolute numbers of circulating nonclassical monocytes strongly correlated with tumor burden $(P<0.0001$, Spearman $r=0.8137$; Supplementary Figure 3D). Notably, the depletion of (non-classical) monocytes in WT recipients significantly delayed tumor development even in the presence of $\mathrm{CD}^{+} \mathrm{T}$ cells. This suggests that in the TCL1-tg mouse, main tumor support may not be derived from $\mathrm{CD}^{+} \mathrm{T}$ cells, but from the monocytic population. Alternatively, it can signify that the loss of monocytes can negatively impact the quality of $\mathrm{CD} 4^{+} \mathrm{T}$ cell support to CLL cells, ${ }^{15}$ highlighting the important interplay between monocytes and T cells in CLL.'

It is striking that the loss of CD4+ T cells in this murine model promotes tumor progression. Several scenarios could contribute to this observation, including the loss of CD4+ Th1 help necessary for effective anti-tumor CTL responses, or the loss of a CD4+ T-cell subset that can negatively modulate monocyte function or has direct anti-tumor activity, such as natural killer T cells. Experiments are underway in order to discriminate between these possibilities.

Taken together, our data demonstrate that CD4+ T cells are dispensable for the establishment and progression of CLL in the TCL1-tg mouse model. Similar to human CLL, monocytes-and in particular the non-classical subset-serve as an important tumorsupportive cell population in the development of TCL1-induced murine CLL.

\section{CONFLICT OF INTEREST}

The authors declare no conflict of interest.

\section{ACKNOWLEDGEMENTS}

We thank Drs G Brachtl and SW Hofbauer for critical reading of the manuscript. This work was supported by the Austrian Academy of Science (DOC Stipendium 23137 to TK), the Austrian Science Fund (Hertha-Firnberg T516-B13 to NZ and T671-B13 to DA; 11299-B12 DACH FOR2036 to AE and SFB021-P11 to RGr), the Paracelsus Medical University Salzburg Research Fund (E-10/11/057-EGL, E-11/13/063-EGL to AE and E-11/14/070-EGP to AE and JPH), DEBRA Austria, 'SCRI-LIMCR GmbH' and the province of Salzburg.

T Kocher $^{1,2}$, D Asslaber ${ }^{1,3}$, N Zaborsky ${ }^{1,3}$, S Flenady ${ }^{1,3}$, U Denk $^{1,3}$, P Reinthaler ${ }^{1,3}$, M Ablinger ${ }^{1}$, R Geisberger ${ }^{1,3}$, JW Bauer ${ }^{2}$, M Seiffert ${ }^{\prime}$, TN Hartmann ${ }^{1,3}$, R Greil ${ }^{1,3}$, A Egle $^{1,3,5}$ and J Piñón Hofbauer ${ }^{1,2,5}$ ${ }^{1}$ Laboratory for Immunological and Molecular Cancer Research, Department of Internal Medicine III with Hematology, Medical Oncology, Hemostaseology, Infectious Diseases, Rheumatology, Oncologic Center, Paracelsus Medical University, Salzburg, Austria;

${ }^{2}$ EB House Austria, Research Program for the Molecular Therapy of Genodermatoses, Department of Dermatology, University Hospital of the Paracelsus Medical University, Salzburg, Austria; ${ }^{3}$ Salzburg Cancer Research Institute, Salzburg, Austria and ${ }^{4}$ Division of Molecular Genetics, German Cancer Research Center (DKFZ), Heidelberg, Germany E-Mail: a.egle@salk.at

${ }^{5}$ These authors contributed equally to this work.

\section{REFERENCES}

1 Zaborsky N, Holler C, Geisberger R, Asslaber D, Gassner FJ, Egger V et al. $B$ cell receptor usage correlates with the sensitivity to CD40 stimulation and the occurence of CD4+ T cell clonality in chronic lymphocytic leukemia. Haematologica 2015; 100: e307-e310.

2 Patten PE, Buggins AG, Richards J, Wotherspoon A, Salisbury J, Mufti GJ et al. CD38 expression in chronic lymphocytic leukemia is regulated by the tumor microenvironment. Blood 2008; 111: 5173-5181.

3 Asslaber D, Grossinger EM, Girbl T, Hofbauer SW, Egle A, Weiss L et al. Mimicking the microenvironment in chronic lymphocytic leukaemia - where does the journey go? Br J Haematol 2013; 160: 711-714.

4 Bagnara D, Kaufman MS, Calissano C, Marsilio S, Patten PE, Simone R et al. A novel adoptive transfer model of chronic lymphocytic leukemia suggests a key role for T lymphocytes in the disease. Blood 2011; 117: 5463-5472.

5 Tinhofer I, Weiss L, Gassner F, Rubenzer G, Holler C, Greil R. Difference in the relative distribution of $\mathrm{CD} 4+\mathrm{T}$-cell subsets in $\mathrm{B}-\mathrm{CLL}$ with mutated and unmutated immunoglobulin (Ig) VH genes: implication for the course of disease. J Immunother 2009; 32: 302-309.

6 Kater AP, Tonino SH, Egle A, Ramsay AG. How does lenalidomide target the chronic lymphocytic leukemia microenvironment? Blood 2014; 124: 2184-2189.

7 McClanahan F, Hanna B, Miller S, Clear AJ, Lichter P, Gribben JG et al. PD-L1 Checkpoint blockade prevents immune dysfunction and leukemia development in a mouse model of chronic lymphocytic leukemia. Blood 2015; 126: 203-211.

8 Han WR, Zhan Y, Murray-Segal LJ, Brady JL, Lew AM, Mottram PL. Prolonged allograft survival in anti-CD4 antibody transgenic mice: lack of residual helper $\mathrm{T}$ cells compared with other CD4-deficient mice. Transplantation 2000; 70: $168-174$.

9 Gorgun G, Ramsay AG, Holderried TA, Zahrieh D, Le DR, Liu F et al. E(mu)-TCL1 mice represent a model for immunotherapeutic reversal of chronic lymphocytic leukemia-induced T-cell dysfunction. Proc Natl Acad Sci USA 2009; 106: 6250-6255.

10 Hofbauer JP, Heyder C, Denk U, Kocher T, Holler C, Trapin D et al. Development of CLL in the TCL1 transgenic mouse model is associated with severe skewing of the T-cell compartment homologous to human CLL. Leukemia 2011; 25: 1452-1458.

11 Maffei R, Bulgarelli J, Fiorcari S, Bertoncelli L, Martinelli S, Guarnotta C et al. The monocytic population in chronic lymphocytic leukemia shows altered composition and deregulation of genes involved in phagocytosis and inflammation. Haematologica 2013; 98: 1115-1123. 
12 Seiffert M, Schulz A, Ohl S, Dohner H, Stilgenbauer S, Lichter P. Soluble CD14 is a novel monocyte-derived survival factor for chronic lymphocytic leukemia cells, which is induced by CLL cells in vitro and present at abnormally high levels in vivo. Blood 2010; 116: 4223-4230.

13 Ziegler-Heitbrock L, Ancuta P, Crowe S, Dalod M, Grau V, Hart DN et al. Nomenclature of monocytes and dendritic cells in blood. Blood 2010; 116: e74-e80.

14 van RN, Hendrikx E. Liposomes for specific depletion of macrophages from organs and tissues. Methods Mol Biol 2010; 605: 189-203.

15 Hanna BS, McClanahan F, Yazdanparast H, Zaborsky N, Kalter V Rößner P et al. Depletion of CLL-associated patrolling monocytes and macrophages controls disease development and repairs immune dysfunction in vivo. Leukemia 2016; 30: 570-579.

c) (1) $\Theta$ This work is licensed under a Creative Commons AttributionNC ND NonCommercial-NoDerivs 4.0 International License. The images or other third party material in this article are included in the article's Creative Commons license, unless indicated otherwise in the credit line; if the material is not included under the Creative Commons license, users will need to obtain permission from the license holder to reproduce the material. To view a copy of this license, visit http:// creativecommons.org/licenses/by-nc-nd/4.0/

Supplementary Information accompanies this paper on the Leukemia website (http://www.nature.com/leu)

\section{OPEN}

\section{Efficacy of ruxolitinib on hepatomegaly in patients with myelofibrosis}

Leukemia (2016) 30, 1413-1415; doi:10.1038/leu.2015.310

The Janus kinase $1 / 2$ inhibitor ruxolitinib has been shown in a pivotal placebo-controlled phase III study (Controlled Myelofibrosis Study with Oral JAK Inhibitor Treatment I (COMFORT-I)) to significantly reduce splenomegaly and symptoms in patients with intermediate-2 or high-risk myelofibrosis (MF). Although splenomegaly is the most common manifestation of MF-associated extramedullary hematopoiesis, up to $65 \%$ of patients may also have hepatomegaly, ${ }^{1,2}$ which is often highly symptomatic and can lead to serious complications. ${ }^{3}$ Here, we present the results from a COMFORT-I post hoc analysis that assessed changes in liver volume and their relationship with concomitant changes in splenomegaly and measures of metabolic status.

As previously reported, ${ }^{4}$ COMFORT-I was a randomized, double-blind, placebo-controlled phase III study in patients with intermediate-2 or high-risk MF and a baseline spleen length $\geqslant 5 \mathrm{~cm}$ below the left costal margin. Patients randomized to placebo were allowed to cross over to ruxolitinib if they met prespecified criteria for disease progression. The primary end point was the proportion of patients who achieved $a \geqslant 35 \%$ reduction in spleen volume at week $24 .{ }^{4}$ Liver and spleen volumes were determined by magnetic resonance imaging or computed tomography per protocol; palpable liver length was not assessed. Percentage changes of spleen and liver volumes from baseline to week 144 were calculated for observed cases. For patients who crossed over from placebo to ruxolitinib, liver volume values obtained after crossover were excluded from the analysis for placebo and analyzed separately. In these individuals, baseline was defined as the last observation before crossover. Percentage changes in liver volume at week 24 were compared between treatment arms by analysis of covariance, with baseline liver volume as the covariate. Correlations of clinical parameters were assessed by Pearson's correlation coefficient with associated test for zero correlation and the $R^{2}$ statistic calculated by simple linear regression. A $5 \%$ significance level was applied to all statistical tests, without adjustment for multiple testing.

A total of 139 of 155 patients randomized to ruxolitinib and 105 of 154 patients randomized to placebo had evaluable liver volume data at baseline and week 24. Median (range) liver volume at baseline was $2452 \mathrm{~cm}^{3}\left(1268-4833 \mathrm{~cm}^{3}\right)$ in the ruxolitinib arm, and $2485 \mathrm{~cm}^{3}\left(1298-5230 \mathrm{~cm}^{3}\right)$ in the placebo arm (see Supplementary Table S1 for additional baseline characteristics). At week 24, patients treated with ruxolitinib had a mean percentage change (decrease) in liver volume of $-7.9 \%$ (median change, $-8.7 \%$; range, $-26.0 \%$ to $+17.5 \%$ ) compared with a mean increase of $3.5 \%$ (median change, $4.1 \%$; range, $36.0 \%$ to $+41.7 \%$ ) in the placebo arm $(P<0.0001$; Figures $1 \mathrm{a}$ and $\mathrm{b})$. The extent of liver volume reductions tended to be greater among patients who achieved average doses of 20 or $25 \mathrm{mg}$ twice daily during weeks 21-24 (mean changes at week $24:-9.7 \%$ and $-9.8 \%$, respectively) than among patients with lower average doses during weeks 21-24 (mean changes: $-5.1 \%$ with $<10 \mathrm{mg}$ twice daily, $-6.0 \%$ with $10 \mathrm{mg}$ twice daily and $-6.5 \%$ with $15 \mathrm{mg}$ twice daily).

In the ruxolitinib arm, liver volumes decreased rapidly during the first 12 weeks and continued to decrease until approximately week 48 , with a mean percentage change of $-10.6 \%$ at week 48 (range, -35.9 to +23.0 ). Improvement was maintained through week 144 (Figure 1c). In contrast, in the placebo group, liver volume increased over time, resulting in a mean percentage change of $6.0 \%$ at week 48 (range, $-21.6 \%$ to $+43.0 \%$ ) when most patients in the placebo group had discontinued or crossed over to ruxolitinib. Patients who crossed over from placebo to ruxolitinib experienced decreases in liver volume from the point of crossover, with an overall time course similar to that observed in patients randomized to ruxolitinib (Figure 1c).

We sought to determine whether the percentage changes in liver volume in the ruxolitinib arm were affected by baseline liver volume. Patients in the first $(n=37)$, second $(n=35)$, third $(n=36)$ and fourth $(n=31)$ quartile had median (range) baseline liver volumes of $1929 \mathrm{~cm}^{3}\left(1268-2115 \mathrm{~cm}^{3}\right), 2323 \mathrm{~cm}^{3}$ $\left(2131-2483 \mathrm{~cm}^{3}\right), \quad 2661 \mathrm{~cm}^{3}\left(2488-3014 \mathrm{~cm}^{3}\right)$ and $3417 \mathrm{~cm}^{3}$ $\left(3028-4833 \mathrm{~cm}^{3}\right)$, respectively. Patients experienced similar reductions in hepatomegaly at week 24 regardless of baseline liver volume, with mean \pm s.d. percentage changes in liver volume of $-8.3 \% \pm 7.9 \%,-6.4 \% \pm 7.5 \%,-7.8 \% \pm 8.6 \%$ and $-9.1 \% \pm 8.8 \%$ in the first, second, third and fourth quartile, respectively. Changes in liver volume over time observed for the entire study population were reflected in each quartile (Figure 1d). Although the mean values for each quartile showed some differences after week 60, the interpatient variability in each quartile (represented by the 